\title{
PERFIL DOS USUÁRIOS DO SERVIÇO DE SAÚDE BUCAL EM COMENDADOR LEVY GASPARIAN/RJ
}

\section{ARTIGO ORIGINAL}

MELO, Renata Patrícia Pereira de ${ }^{1}$

SILVA, Natália Leal da ${ }^{2}$

MACHADO, Fátima Lúcia Cartaxo ${ }^{3}$

SOUZA, Rafael de ${ }^{4}$

MELO, Renata Patrícia Pereira de. Et al. Perfil dos usuários do serviço de saúde bucal em Comendador Levy Gasparian/RJ. Revista Científica Multidisciplinar Núcleo do Conhecimento. Ano 05, Ed. 10, Vol. 07, pp. 73-91. Outubro de 2020. ISSN: 2448-0959, Link de acesso: https://www.nucleodoconhecimento.com.br/odontologia/saude-bucal

\section{RESUMO}

A saúde dos dentes e da cavidade oral afeta a qualidade de vida das pessoas, pois interfere na capacidade de comer, comunicar-se, na estética e na prevenção de outras doenças. O objetivo da pesquisa é identificar o perfil dos usuários dos serviços de saúde bucal das unidades básicas de saúde do município de Comendador Levy Gasparian/RJ. A população do estudo foi composta por todos os usuários do serviço odontológico do município e o tamanho da amostra para coleta utilizou uma

\footnotetext{
${ }^{1}$ Graduanda em Administração Pública (UFF/PUVR).

2 Graduanda em Administração Pública (UFF/PUVR).

${ }^{3}$ Bel $^{a}$ em Odontologia e Mestranda em Ciências Ambientais (Universidade de Vassouras).

${ }^{4}$ Me. em Administração (UFF/PPGAd), Bel. em Administração Pública (UFF/PUVR), graduando em Ciência de Dados (UNIVESP).
} 
amostragem não paramétrica do tipo por conveniência. A pesquisa foi executada usando a abordagem quantitativa e a análise dos dados por meio da Estatística Descritiva. Para facilitar a análise e compressão foram construídos gráficos com dados univariados e bivariados. Os resultados demonstraram as mulheres, os jovens, os idosos, pessoas da raça amarela e pacientes com Ensino Superior representam grupos que menos usam os serviços odontológicos do município e estratégias que atraiam tais públicos podem aumentar o acesso ao serviço. As unidades próximas ao centro urbano do município receberam um número maior de participantes.

Palavras-chave: Saúde Pública, Atenção Primária à Saúde, saúde bucal, análise estatística.

\section{INTRODUÇÃO}

As mudanças sociais e geográficas impuseram desafios à reestruturação dos sistemas e aos serviços de saúde em muitos países do mundo nas últimas décadas e alguns deles continuam em mudanças até o presente.

Desde o final do século $X X$ a saúde pública e os serviços de saúde pública enfrentam desafios sem precedentes. Os países se depararam com vários desafios à saúde, dentre os quais incluem doenças transmissíveis e não-transmissíveis.

A importância de promover um comportamento saudável e abordar os determinantes sociais e econômicos que tornam a saúde precária é destacada pela incidência e pela topografia social de doenças não-transmissíveis e, em muitos casos, das classificadas como transmissíveis.

O objetivo tradicional da saúde pública de proteger a saúde do público, principalmente por meio de ações preventivas de doenças e proteção e promoção da saúde, tornase mais evidente do que nunca.

A governança da saúde pública é algo complexo e composta por multifacetas, pois a organização e a prestação de serviços públicos de saúde envolvem muitas instituições e profissões distintas. 
Os programas, serviços, atividades que compõem a saúde pública são amplos e variados no Brasil. Em especial, na atenção primária da saúde pública existem diversos princípios, diretrizes, políticas, programas, projetos e estruturas organizacionais que representam medidas públicas de promoção da saúde, prevenção, tratamento e reabilitação da saúde dos usuários.

Uma política pública brasileira que se dedica à atenção da saúde bucal é denominada Política Nacional de Saúde Bucal. A partir desta política, o Brasil passou a figurar, segundo a Organização Mundial de Saúde (OMS), um seleto grupo de países considerados com baixa prevalência de cáries.

Como parte integrante da Política Nacional de Saúde Bucal, foi instituído o Programa Brasil Sorridente. O objetivo do programa é prever ações que facilitem e ampliem o acesso dos usuários do Sistema Único de Saúde aos tratamentos odontológicos gratuitos em diferentes municípios brasileiros.

A saúde dos dentes e da cavidade oral afeta a qualidade de vida das pessoas, pois interferem na capacidade diária de comer, falar e manter a comunicação. O tratamento odontológico é forma de prevenção de doenças, eliminação da dor e desconforto na região. Além da intervenção direta na região, cabe destacar que a literatura indica a existência de uma relação entre aspectos da saúde bucal e a contribuição para o surgimento de outras doenças, tais como diabetes, doenças cardiovasculares, câncer e outras.

Dados e informação do Instituto Nacional do Câncer alertam para a necessidade de atenção à saúde bucal. De acordo com o referido instituto, a estimativa de incidência de câncer de boca atingirá aproximadamente 15 mil pessoas para cada ano do triênio 2020-2022. Daquele total, cerca de 11,1 mil pessoas serão do sexo masculino enquanto 4 mil em mulheres (BRASIL, 2019).

Frente ao exposto, a presente pesquisa tem por objetivo identificar o perfil dos usuários dos serviços de saúde bucal prestados pelas unidades básicas de saúde do município de Comendador Levy Gasparian/RJ. 
Para alcançar o objetivo da pesquisa, foi construído um questionário do tipo estruturado no formato impresso para coleta de dados junto aos usuários nas unidades básicas de saúde do município.

Após a aplicação dos instrumentos de pesquisa, aos dados foram utilizadas técnicas de estatística descritiva para facilitar a visualização e interpretação dos resultados.

Os pesquisadores também esperam que os dados referentes às características demográficas, colhidos nas respostas do instrumento de pesquisa, indiquem os grupos específicos que por ventura necessitariam de ações direcionadas.

O artigo está estruturado nos capítulos referente a esta Introdução e no Referencial teórico dividido em quatro subtítulos: Atenção primária à saúde, Saúde bucal, Política Nacional de Saúde Bucal e Avaliação de serviços de saúde. Posteriormente, o capítulo da metodologia apresenta a estratégia adotada na pesquisa. Os capítulos referentes as discussões, resultados e conclusões foram desenvolvidos após o período de coleta dos dados. Por fim, as referências bibliográficas que contribuíram para a construção do artigo foram listadas no último capítulo.

\section{REVISÃO DA LITERATURA}

\subsection{ATENÇÃO PRIMÁRIA À SAÚDE}

A mudança das economias planejadas para as de mercado tem sido o principal motor das reformas do sistema de saúde, que na maioria dos países se mostraram insuficientes para manter programas preventivos adequados, como a vacinação infantil, e fornecer o acesso universal aos serviços básicos de saúde.

Os serviços públicos de saúde têm um papel vital a desempenhar no enfrentamento aos desafios de saúde pelo globo. A Organização Mundial da Saúde (OMS) incentiva aos Estados Membros a colocarem o fortalecimento de seu sistema de saúde no centro das políticas e ações intergovernamentais, adotando planos e estratégias bem definidos. 
A OMS procura ajudar os Estados Membros a fortalecer e a melhorar os seus serviços públicos de saúde, como parte vital do sistema de saúde e foco na prevenção de doença, assim como na proteção e promoção da saúde.

Quadro 1: Níveis de atenção à Saúde

\begin{tabular}{|c|c|c|}
\hline Níveis & Definição & Exemplos \\
\hline Primária & $\begin{array}{l}\text { Medidas de promoção da saúde, } \\
\text { prevenção, tratamento e } \\
\text { reabilitação. Os procedimentos } \\
\text { envolvem tecnologias de baixo } \\
\text { custo. }\end{array}$ & $\begin{array}{l}\text { Atendimentos de rotina: } \\
\text { consultas com o clínico geral, } \\
\text { tratamentos, vacinação, pré- } \\
\text { natal, atendimento odontológico } \\
\text { e acompanhamento de } \\
\text { hipertensos e diabéticos. }\end{array}$ \\
\hline Secundária & $\begin{array}{l}\text { Consiste no tratamento da doença } \\
\text { já instalada, visando evitar o } \\
\text { aparecimento de deficiências ou } \\
\text { incapacidades. São formados pelos } \\
\text { serviços especializados em nível } \\
\text { ambulatorial e hospitalar, com } \\
\text { densidade } \\
\text { intermediária. }\end{array}$ & $\begin{array}{l}\text { Serviços } r \text { médicos } \\
\text { especializados, de apoio } \\
\text { diagnóstico e terapêutico e } \\
\text { atendimento de urgência e } \\
\text { emergência. }\end{array}$ \\
\hline Terciária & $\begin{array}{l}\text { Designa o conjunto de terapias e } \\
\text { procedimentos de elevada } \\
\text { especialização. Organiza também } \\
\text { procedimentos que envolvem alta } \\
\text { tecnologia e/ou alto custo. }\end{array}$ & $\begin{array}{l}\text { Procedimentos ambulatoriais } \\
\text { de alta complexidade estão a } \\
\text { quimioterapia, a radioterapia, a } \\
\text { hemoterapia, a ressonância } \\
\text { magnética e a medicina } \\
\text { nuclear. }\end{array}$ \\
\hline
\end{tabular}

Fonte: Adaptado de Ministério da Saúde (2017). (Elaborado pelos autores).

A Atenção Primária à Saúde corresponde à assistência necessária e acessível a cada pessoa de forma individual, além ser aplicada à população como um todo. Ela é fornecida de forma aceitável aos usuários, para participarem de forma ativa e com os 
custos correspondentes às capacidades da população e do Estado (MINISTÉRIO DA SAÚDE, 2017).

A Atenção Primária à Saúde é um dos critérios para uso racional de todo o sistema público de saúde brasileiro. O objetivo dela é solucionar os principais problemas de saúde pública, através de medidas de promoção da saúde, prevenção, tratamento e reabilitação. A lógica da estratégia se baseia em investir mais na prevenção primária, para reduzir o custo com atendimentos às vítimas e maior o impacto e a abrangência na proteção da população (MINISTÉRIO DA SAÚDE, 2017).

Em virtude das condições econômicas, dos valores sociais, das características geográficas e da cultura da atenção primária à saúde em várias localidades pode ter características específicas, mas, independentemente disso, inclui a seguinte função.

A Atenção Primária à Saúde foi desenvolvida para promover justiça social e equidade ao reconhecer a saúde como um direito fundamental, consoante ao artigo $25 \mathrm{da}$ Declaração Universal dos Direitos Humanos que defende que os seres humanos têm "[...] direito a um padrão de vida capaz de assegurar a si e a sua família saúde e bem estar, inclusive alimentação, vestuário, habitação, cuidados médicos e os serviços sociais indispensáveis [...]" (ONU, 2009).

No ano de 2018, representantes de diferentes nações reafirmaram o compromisso de fortalecer à atenção primária à saúde por meio da Declaração de Astana. Este documento propõe que os Estados busquem alcançar à cobertura universal de saúde e representa um elemento de abordagem fundamental para alcançar os Objetivos de Desenvolvimento Sustentável (ODS) relacionados à saúde (GIOVANELLA et al., 2019).

O Quadro 2 demonstra os princípios básicos da Atenção Primária à Saúde defendidos pelo Ministério da Saúde.

Quadro 2: Princípios básicos da Atenção Primária à Saúde

Princípios Definição




\begin{abstract}
Universalidade Possibilitar o acesso universal e contínuo a serviços de saúde de qualidade e resolutivos, caracterizados como a porta de entrada aberta e preferencial da Rede de Atenção à Saúde (primeiro contato), acolhendo as pessoas e promovendo a vinculação e responsabilização solidária pela atenção às suas necessidades de saúde.

Equidade Ofertar o cuidado, reconhecendo as diferenças nas condições de vida e saúde e de acordo com as necessidades das pessoas, considerando que o direito à saúde passa pelas diferenciações sociais e deve atender à diversidade.

Integralidade É o conjunto de serviços executados pela equipe de saúde que atendam às necessidades da população adstrita nos campos do cuidado, da promoção e manutenção da saúde, da prevenção de doenças e agravos, da cura, da reabilitação, redução de danos e dos cuidados paliativos.
\end{abstract}

Fonte: Adaptado de Ministério da Saúde (2017). (Elaborado pelos autores).

Os princípios básicos orientam um conjunto de iniciativas do Ministério da Saúde que atende aos usuários no ambiente em que vivem. Dentre outros programas, ações e estratégias de atenção primária à saúde, faz-se relevante destacar a existência de algumas iniciativas: (a) Política Nacional de Atenção Básica; (b) Política Nacional de Atenção Integral à Saúde das Pessoas Privadas de Liberdade no Sistema Prisional; (c) Política Nacional de Alimentação e Nutrição; (d) Política Nacional de Saúde Bucal; (e) Programa Telessaúde Brasil Redes; (f) Programa Nacional de Melhoria do Acesso e Qualidade na Atenção Básica (PMAQ-AB) e outros.

Dentre as iniciativas anteriores, a Política Nacional de Saúde Bucal é aplicada à Atenção Primária à Saúde ao promover ações de promoção, prevenção e recuperação da saúde bucal da população por meio da rede de unidades básicas do Sistema Único de Saúde. 


\subsection{SAÚDE BUCAL}

Os termos saúde bucal e geral não devem ser interpretados como conceitos separados. A saúde bucal é parte integrante da saúde de todos e do bem-estar de todas as pessoas.

Além da questão da saúde, problemas de ordem odontológicos podem ter consequências sociais, tais como transtorno de personalidade em função de comentários vexatórios de terceiros e que podem resultar problemas relacionados a estima e alienação social, são aspectos que resultam do perfil de impacto da saúde bucal (SLADE; SPENCER, 1994; FERREIRA et al., 2020).

A Organização Mundial de Saúde define a saúde bucal como um estado livre de dores bucais e faciais, doenças e distúrbios bucais que limitam a capacidade de um indivíduo de morder, mastigar, sorrir, falar e bem-estar psicossocial (WHO, 2013).

A região bucal abrange a cavidade oral e não se restringe os dentes, gengivas e tecidos de suporte, mas também o palato, a membranas moles e mucosas da boca e laringe, língua, lábios, glândulas salivares, músculos mastigatórios e mandíbula (WHO, 2013).

A saúde bucal é essencial para a saúde e o bem-estar em geral, visto que ela influencia a qualidade de vida. A detecção oportuna de doenças bucais pode contribuir para o diagnóstico e o tratamento de várias doenças sistêmicas (ARAÚJO-JÚNIOR et al., 2018; SOUZA et al., 2018).

Os problemas típicos de saúde bucal são as cáries dentárias, doenças periodontais, mal oclusão, ferimento, câncer bucal e outros. Um exame minucioso da cavidade oral revela sinais de deficiência de nutrientes, além de diversas doenças sistêmicas, incluindo as infecções, os distúrbios imunológicos, as lesões e certos tipos de cânceres (SANTOS et al., 2011; TERRA; GOULART; BAVARESCO, 2011; WHO, 2013; OLIVEIRA, 2018). 
O câncer da boca é um tumor maligno que pode afeta os lábios e a estruturas da boca (gengivas, bochechas, céu da boca, língua e a região embaixo da língua). É comum em homens acima dos 40 anos, figura como o quarto tipo de tumor presentes em pessoas do sexo masculino na região Sudeste. A maioria dos casos é diagnosticada em estágios avançados (GUERRA; SANTOS, 2018; MINISTÉRIO DA SAÚDE, 2019).

Entre os anos de 2007 e 2017, foram registrados 26.510 de óbitos por cânceres no lábio, na base da língua, em outras partes da língua, na gengiva, no assoalho da boca, no palato e em outras partes da boca (INCA, [?]).

A Organização Mundial da Saúde estima que a prevenção pode ajudar na redução da incidência de casos de câncer em aproximadamente $25 \%$ até o ano de 2025 . A visita periódica ao dentista favorece ao diagnóstico precoce do câncer de boca, já que permite identificar as lesões classificadas como suspeitas.

Diagnosticados no início e tratados da maneira adequada, a maioria dos casos desse tipo de câncer tem curas: $80 \%$ das incidências. Geralmente, o tratamento envolve cirurgia oncológica e/ou radioterapia. A avaliação médica, conforme cada caso, vai decidir a forma de tratamento mais adequada (MINISTÉRIO DA SAÚDE, 2018; OLIVEIRA et al., 2018).

Algumas doenças podem afetar a saúde bucal e exigem um tratamento multidisciplinar, como diabetes e vírus do HIV. Pessoas com diabetes têm maior risco de desenvolver doenças bucais causadas por várias bactérias, vírus e fungos. Altos níveis de açúcar na saliva podem desencadear bactérias na boca. $O$ diabetes também pode causar uma diminuição na quantidade de saliva, que por sua vez pode causar doenças gengivais e cáries (TERRA; GOULART; BAVARESCO, 2011; SOUZA et al., 2018; JUCÁ et al, 2019).

O vírus da imunodeficiência pode causar cáries dentárias, doenças gengivais e infecções bucais, como papilomas orais, infecções fúngicas e úlceras. Os medicamentos usados contra o do vírus HIV podem causar boca seca (BRASIL, 2004; SANTOS et al., 2011; JUCÁ et al, 2019). 
Dadas as dimensões físicas e sociais relacionadas à saúde bucal, o setor público brasileiro desenvolveu uma política pública nacional direcionada.

\subsection{POLÍTICA NACIONAL DE SAÚDE BUCAL}

A Política Nacional de Saúde Bucal se constitui como um marco na história das políticas públicas brasileira ao incorporar o tema uma agenda em discussão desde o Movimento pela Reforma Sanitária Brasileira (BRASIL, 2004; BRASIL, 2013).

A principal meta estipulada na Política Nacional de Saúde Bucal prevê a reorganização da prática e a qualificação das ações e serviços oferecidos no país, reunindo medidas para garantir ações de promoção, prevenção e recuperação da saúde bucal dos brasileiros de todas as idades, com acesso ampliado aos serviços relativos ao tratamento odontológico gratuito por meio do SUS (BRASIL, 2004).

Como parte da Política Nacional de Saúde Bucal, o Programa Brasil Sorridente do governo federal reúne uma série de medidas para garantir ações de promoção, prevenção e recuperação da saúde bucal dos brasileiros. Com isso, o programa busca facilitar e ampliar o acesso da população ao tratamento odontológico gratuito aos brasileiros por meio do Sistema Único de Saúde (SUS) (BRASIL, 2004; BRASIL, 2013; PERES et al., 2012).

Antes do Brasil Sorridente, o País não tinha política pública de saúde para o setor. Apenas quem podia pagar por uma consulta tinha cesso a atendimento odontológico no Brasil. Porém, antes do Brasil Sorridente, praticamente não se efetivava a reabilitação em saúde bucal (BRASIL, 2004).

Ao trabalhar os eixos da atenção à saúde bucal a partir do incremento da atenção básica por meio da Estratégia Saúde da Família, da implementação dos Centros de Especialidades Odontológicas como elemento estruturante da atenção secundária, além das ações de caráter coletivo, o Programa Brasil Sorridente se insere no conjunto de programas estratégicos da atual política de saúde (BRASIL, 2013). 
A política nacional direcionada aos serviços odontológicos em unidades públicas de saúde permitiu diminuir as desigualdades sociais no acesso e tratamentos da saúde bucal, visto que expandiu a oferta dos serviços em diferentes municípios brasileiros (PERES et al., 2012).

\section{MÉTODOS}

A pesquisa se classifica como exploratória, seleção da abordagem quantitativa, utilização da Estatística descritiva (univariada e bivariada) e a coleta dos dados por meio questionário do tipo estruturado impresso e com questões objetivas correspondentes ao aspecto demográfico (BÊRNI; FERNANDEZ, 2012; CRESWELL, 2010).

A população do estudo considerou todos os usuários dos serviços de saúde bucal do município e a amostra da pesquisa foi por meio da amostragem não probabilística do tipo amostragem por conveniência, visto que tal técnica que permite maior grau de liberdade ao pesquisador na definição da quantidade de participantes (BÊRNI; FERNANDEZ, 2012).

Apesar do questionário impresso ser custoso, tal escolha teve o intuito de atingir uma parcela maior de participantes, ao passo que o uso de questionário online poderia ser um limitador em virtude das características econômica e regional da cidade (BÊRNI; FERNANDEZ, 2012).

O instrumento de pesquisa foi estruturado em um bloco, no qual foram distribuídas as assertivas que buscaram identificar o perfil demográfico dos participantes: faixa etária, gênero, raça, escolaridade e unidade de saúde que frequenta.

Os dados coletados foram tabulados em uma planilha, ao passo que o tratamento e as análises ocorreram o auxílio do software $R$ Studio. 


\section{RESULTADOS}

A pesquisa foi realizada nas unidades básicas de saúde do município de Comendador Levy Gasparian, situado na região Centro Sul Fluminense do Estado do Rio de Janeiro. A coleta de dados ocorreu no período de 15 de novembro a 15 de dezembro de 2019.

Os dados iniciais demonstraram que a base de dados inicial era composta por 18 variáveis (colunas) e 118 observações (linhas). $\mathrm{Na}$ análise exploratória, foram identificados dados faltantes em virtude das assertivas que não foram preenchidas pelos respondentes.

No intuito de viabilizar a análise, os dados faltantes foram suprimidos e uma nova base de dados foi gerada, composta pelas 18 variáveis e 106 observações, com uma perda de $10,16 \%$ dos dados.

A análise demográfica dos dados dos respondentes foi realizada de forma univariada e bivariada.

Em relação a faixa etária, a análise univariada demonstra que a maioria dos usuários se concentram entre 31 e 60 anos. Observa-se que após os 40 anos a quantidade de pacientes diminui, particularmente na população idosa, assim como na população mais jovem.

Tabela 1 - Faixa etária

\begin{tabular}{|l|l|}
\hline Faixa etária & Quantidade \\
\hline 18 a 20 anos & 9 \\
\hline 21 a 30 anos & 10 \\
\hline 31 a 40 anos & 32 \\
\hline 41 a 50 anos & 22 \\
\hline 51 a 60 anos & 22 \\
\hline 61 a 70 anos & 9 \\
\hline
\end{tabular}




\section{Mais de 70 anos 1 \\ Prefiro não informar 1}

Fonte: Resultado da pesquisa (Elaborado pelos autores).

A Tabela 2 demonstra uma quantidade expressiva de pacientes do gênero masculino (82\%) em comparação ao feminino (17\%).

Tabela 2: Gênero

\begin{tabular}{|l|l|}
\hline Gênero & Quantidade \\
\hline Masculino & 87 \\
\hline Feminino & 18 \\
\hline Prefiro não informar & 1 \\
\hline
\end{tabular}

Fonte: Resultado da pesquisa (Elaborado pelos autores).

Os pacientes que se declararam como pardos representam a maior parcela dos usuários dos serviços de odontologia no município pesquisado, ao passo que pacientes da raça amarela representa a minoria da população. Destaca-se que os quantitativos de pacientes das raças branca e negra são próximos.

Tabela 3 - Raça

\begin{tabular}{|l|l|}
\hline Raça & Quantidade \\
\hline Amarelo & 3 \\
\hline Branco & 33 \\
\hline Negro & 28 \\
\hline Pardo & 38 \\
\hline Prefiro não informar & 4 \\
\hline
\end{tabular}

Fonte: Resultado da pesquisa (Elaborado pelos autores). 
Os usuários do serviço de saúde bucal com escolaridade nos níveis médio (54) e fundamental (40) representam maioria, correspondendo respectivamente em termos percentuais a $51 \%$ e $38 \%$ dos respondentes.

Tabela 4 - Escolaridade

10Prefiro não informar2

\begin{tabular}{|l|l|}
\hline Escolaridade & Quantidade \\
\hline Ensino Fundamental & 40 \\
\hline Ensino Médio & 54 \\
\hline Ensino Superior & \\
\hline
\end{tabular}

Fonte: Resultado da pesquisa (Elaborado pelos autores).

A unidade básica de saúde Centro (30) é o órgão que mais teve respondentes quanto aos serviços de odontologia, seguida pela unidade Gulf (26) que se localiza próximo ao centro do município, como apresentado na Tabela 5.

Uma menor adesão por parte dos participantes da unidade básica de Mont Serrat (4) pode decorrer pelo fato da localidade se encontrar em uma zona rural.

Tabela 5 - Unidades básicas de saúde da pesquisa

\begin{tabular}{|l|l|}
\hline Nome da Unidade Básica de Saúde & Quantidade \\
\hline Afonso Arinos & 11 \\
\hline Centro & 30 \\
\hline Conjunto Luiz Bento Argon & 1 \\
\hline Fonseca Almeida & 14 \\
\hline Grotão & 20 \\
\hline Gulf & 26 \\
\hline Mont Serrat & 4 \\
\hline
\end{tabular}

Fonte: Resultado da pesquisa (Elaborado pelos autores). 
A análise bivariada dos dados permite observá-los a partir de duas variáveis e entender por outra perspectiva o contexto (CRESWELL, 2010).

A análise das variáveis faixa etária e gênero permite identificar que o público feminino entre 31 e 50 anos (29\%; 17\%) e o público masculino entre 51 a 60 (7\%) anos, são os usuários que mais utilizam os serviços odontológicos no município. Cabe salientar a ausência de pessoas do gênero masculino com mais de 70 anos (0\%).

Tabela 6 - Proporção entre a faixa etária e o gênero

\begin{tabular}{|l|l|l|l|}
\hline Faixa etária & Fênero & & \\
\hline $\mathbf{1 8}$ a $\mathbf{2 0}$ anos & $6 \%$ & $3 \%$ & $0 \%$ \\
\hline $\mathbf{2 1}$ a $\mathbf{3 0}$ anos & $8 \%$ & $2 \%$ & $0 \%$ \\
\hline $\mathbf{3 1}$ a $\mathbf{4 0}$ anos & $29 \%$ & $1 \%$ & $0 \%$ \\
\hline $\mathbf{4 1}$ a $\mathbf{5 0}$ anos & $17 \%$ & $3 \%$ & $1 \%$ \\
\hline $\mathbf{5 1}$ a $\mathbf{6 0}$ anos & $14 \%$ & $7 \%$ & $0 \%$ \\
\hline $\mathbf{6 1}$ a 70 anos & $7 \%$ & $2 \%$ & $0 \%$ \\
\hline Mais de 70 anos & $1 \%$ & $0 \%$ & $0 \%$ \\
\hline Prefiro não informar & $1 \%$ & $0 \%$ & $0 \%$ \\
\hline
\end{tabular}

Fonte: Resultado da pesquisa (Elaborado pelos autores).

A Tabela 7 demonstra a distribuição proporcional dos dados das variáveis faixa etária e raça dos respondentes.

Pessoas da raça amarela, fora da faixa etária de 41 a 60 anos, possui resultados iguais a $0 \%$.

Usuários das raças branca e negra variam entre 3\% e 9\% nas faixas etárias da pesquisa, exceto para pacientes com mais de 70 anos (0\%). Os pacientes da raça parda são os que se distribuem em todas as faixas etárias, concentrados predominantemente entre 31 e 50 anos (11\%; 12\%). 
Tabela 7 - Proporção entre a faixa etária e a raça

\begin{tabular}{|c|c|c|c|c|c|}
\hline \multirow[b]{2}{*}{ Faixa etária } & \multicolumn{5}{|l|}{ Raça } \\
\hline & Amarelo & Branco & Negro & Pardo & Prefiro não informar \\
\hline 18 a 20 anos & $0 \%$ & $3 \%$ & $4 \%$ & $2 \%$ & $0 \%$ \\
\hline 21 a 30 anos & $0 \%$ & $5 \%$ & $1 \%$ & $4 \%$ & $0 \%$ \\
\hline 31 a 40 anos & $0 \%$ & $9 \%$ & $9 \%$ & $11 \%$ & $0 \%$ \\
\hline 41 a 50 anos & $1 \%$ & $5 \%$ & $2 \%$ & $12 \%$ & $1 \%$ \\
\hline 51 a 60 anos & $2 \%$ & $7 \%$ & $8 \%$ & $5 \%$ & $0 \%$ \\
\hline 61 a 70 anos & $0 \%$ & $3 \%$ & $3 \%$ & $1 \%$ & $2 \%$ \\
\hline Mais de 70 anos & $0 \%$ & $0 \%$ & $0 \%$ & $1 \%$ & $0 \%$ \\
\hline $\begin{array}{l}\text { Prefiro não } \\
\text { informar }\end{array}$ & $0 \%$ & $0 \%$ & $0 \%$ & $0 \%$ & $1 \%$ \\
\hline
\end{tabular}

Fonte: Resultado da pesquisa (Elaborado pelos autores).

Pacientes que de todas as faixas etárias e que concluíram o Ensino Fundamental utilizam os serviços odontológicos do município. Em relação nível de escolaridade de Ensino Médio não foram identificados pacientes com mais de 70 anos.

No grupo de usuários que possuem nível superior, a faixa etária se encontra entre 21 e 60 anos, ou seja, não participaram pacientes mais jovens e idosos.

Tabela 8 - Proporção entre a faixa etária e a escolaridade

\begin{tabular}{|l|l|l|l|l|}
\hline Faixa etária & $\begin{array}{l}\text { Escolaridade } \\
\text { Funsino }\end{array}$ & $\begin{array}{l}\text { Ensino } \\
\text { Médio }\end{array}$ & $\begin{array}{l}\text { Ensino } \\
\text { Superior }\end{array}$ & $\begin{array}{l}\text { Prefiro não } \\
\text { informar }\end{array}$ \\
\hline $\mathbf{1 8}$ a $\mathbf{2 0}$ anos & $1 \%$ & $7 \%$ & $0 \%$ & $1 \%$ \\
\hline $\mathbf{2 1}$ a $\mathbf{3 0}$ anos & $2 \%$ & $6 \%$ & $2 \%$ & $0 \%$ \\
\hline $\mathbf{3 1}$ a $\mathbf{4 0}$ anos & $12 \%$ & $16 \%$ & $2 \%$ & $0 \%$ \\
\hline $\mathbf{4 1}$ a $\mathbf{5 0}$ anos & $8 \%$ & $10 \%$ & $3 \%$ & $0 \%$ \\
\hline $\mathbf{5 1}$ a $\mathbf{6 0}$ anos & $10 \%$ & $8 \%$ & $3 \%$ & $0 \%$ \\
\hline
\end{tabular}




\begin{tabular}{|c|c|c|c|c|}
\hline 61 a 70 anos & $4 \%$ & $5 \%$ & $0 \%$ & $0 \%$ \\
\hline $\begin{array}{l}\text { Mais de } 70 \\
\text { anos }\end{array}$ & $1 \%$ & $0 \%$ & $0 \%$ & $0 \%$ \\
\hline $\begin{array}{l}\text { Prefiro não } \\
\text { informar }\end{array}$ & $0 \%$ & $0 \%$ & $0 \%$ & $1 \%$ \\
\hline
\end{tabular}

Fonte: Resultado da pesquisa (Elaborado pelos autores).

A distribuição da faixa etária dos pacientes por unidades básicas de saúde do município possibilita identificar quais merecem atenção por parte dos gestores.

A unidade Conjunto Luiz Alberto Argon teve baixa adesão, pois apenas 1\% está entre 31 e 40 anos, ao passo que na unidade Centro os dados dos pacientes estão distribuídos em todas as faixas etárias.

A unidade básica de Afonso Arinos não teve participantes na faixa entre 18 a 20 anos e de pacientes com mais de 70 anos. Algo parecido ocorre na unidade Mont Serrat em relação ao público mais jovens e idoso, diferindo apenas pelo fato de pacientes com mais de 61 anos não utilizarem.

Ao observar os dados da unidade Fonseca Almeida, identifica-se que pessoas idosas é um público que não utiliza os serviços odontológicos.

Tabela 9 - Proporção entre a faixa etária e as Unidades Básicas de Saúde

\begin{tabular}{|l|l|l|l|l|l|l|l|}
\hline \multicolumn{10}{|l|}{ Unidade Básica de Saúde } \\
\hline $\begin{array}{l}\text { Faixa } \\
\text { etária }\end{array}$ & $\begin{array}{l}\text { Afonso } \\
\text { Arinos }\end{array}$ & Centro & $\begin{array}{l}\text { Conjunto Luiz } \\
\text { Alberto Argon }\end{array}$ & $\begin{array}{l}\text { Fonseca } \\
\text { Almeida }\end{array}$ & Grotão & Gulf & $\begin{array}{l}\text { Mont } \\
\text { Serrat }\end{array}$ \\
\hline $\begin{array}{l}\mathbf{1 8} \text { a } \\
\text { anos }\end{array}$ & $0 \%$ & $2 \%$ & $0 \%$ & $4 \%$ & $1 \%$ & $2 \%$ & $0 \%$ \\
\hline $\begin{array}{l}\mathbf{2 1} \text { a } \\
\text { anos }\end{array}$ & $1 \%$ & $1 \%$ & $0 \%$ & $3 \%$ & $1 \%$ & $3 \%$ & $1 \%$ \\
\hline
\end{tabular}




\begin{tabular}{|l|l|l|l|l|l|l|l|}
\hline $\begin{array}{l}31 \text { a } \\
\text { anos }\end{array}$ & $4 \%$ & $8 \%$ & $1 \%$ & $2 \%$ & $3 \%$ & $13 \%$ & $0 \%$ \\
\hline $\begin{array}{l}\mathbf{4 1} \text { a } \\
\text { anos }\end{array}$ & $2 \%$ & $8 \%$ & $0 \%$ & $3 \%$ & $6 \%$ & $2 \%$ & $1 \%$ \\
\hline $\begin{array}{l}\mathbf{5 1} \text { a } \\
\text { anos }\end{array}$ & $3 \%$ & $6 \%$ & $0 \%$ & $1 \%$ & $6 \%$ & $4 \%$ & $2 \%$ \\
\hline $\begin{array}{l}\mathbf{6 1} \text { a } \\
\text { anos }\end{array}$ & $1 \%$ & $4 \%$ & $0 \%$ & $0 \%$ & $3 \%$ & $1 \%$ & $0 \%$ \\
\hline $\begin{array}{l}\text { Mais de } \\
\mathbf{7 0} \text { anos }\end{array}$ & $0 \%$ & $1 \%$ & $0 \%$ & $0 \%$ & $0 \%$ & $0 \%$ & $0 \%$ \\
\hline $\begin{array}{l}\text { Prefiro } \\
\text { não }\end{array}$ & $0 \%$ & $0 \%$ & $0 \%$ & $1 \%$ & $0 \%$ & $0 \%$ & $0 \%$ \\
\hline informar & & & & & & & \\
\hline
\end{tabular}

Fonte: Resultado da pesquisa (Elaborado pelos autores).

Pacientes do sexo feminino das raças branca, negra e parda concentram a maioria dos usuários, enquanto homens da raça amarela são a minoria nos consultórios de tratamento odontológico.

Tabela 10 - Proporção entre a gênero e a raça

\begin{tabular}{|l|l|l|l|l|l|}
\hline & \multicolumn{2}{|l}{ Raça } & & & \\
\hline Gênero & Amarelo & Branco & Negro & Pardo & $\begin{array}{l}\text { Prefiro não } \\
\text { informar }\end{array}$ \\
\hline Feminino & $1 \%$ & $25 \%$ & $23 \%$ & $32 \%$ & $2 \%$ \\
\hline Masculino & $2 \%$ & $7 \%$ & $4 \%$ & $4 \%$ & $1 \%$ \\
\hline Prefiro não informar & $0 \%$ & $0 \%$ & $0 \%$ & $0 \%$ & $1 \%$ \\
\hline
\end{tabular}

Fonte: Resultado da pesquisa (Elaborado pelos autores).

A Tabela 11 demonstra que pessoas com Ensino Superior representa o menor grupo, composto por $8 \%$ de mulheres e apenas $2 \%$ de homens. 
Tabela 11 - Proporção entre a gênero e a escolaridade

\begin{tabular}{|l|l|l|l|l|}
\hline Gênero & $\begin{array}{l}\text { Escolaridade } \\
\text { Ensino }\end{array}$ & $\begin{array}{l}\text { Ensino } \\
\text { Fundamental }\end{array}$ & $\begin{array}{l}\text { Ensino } \\
\text { Superior }\end{array}$ & $\begin{array}{l}\text { Prefiro não } \\
\text { informar }\end{array}$ \\
\hline Feminino & $31 \%$ & $42 \%$ & $8 \%$ & $1 \%$ \\
\hline $\begin{array}{l}\text { Masculino } \\
\text { Prefiro não }\end{array}$ & $0 \%$ & $8 \%$ & $2 \%$ & $1 \%$ \\
informar
\end{tabular}

Fonte: Resultado da pesquisa (Elaborado pelos autores).

A unidade Centro concentra o maior percentual do público masculino (7\%), enquanto as unidades Centro (21\%) e Gulf (21\%) atendem a maioria das mulheres.

Tabela 12 - Gênero e as Unidades Básicas de Saúde

\begin{tabular}{|l|l|l|l|l|l|l|l|}
\hline \multicolumn{7}{|l|}{ Unidade Básica de Saúde } \\
\hline Gênero & $\begin{array}{l}\text { Afonso } \\
\text { Arinos }\end{array}$ & $\begin{array}{l}\text { Centro } \\
\text { Conjunto } \\
\text { Luiz Alberto } \\
\text { Argon }\end{array}$ & $\begin{array}{l}\text { Fonseca } \\
\text { Almeida }\end{array}$ & Grotão & Gulf & $\begin{array}{l}\text { Mont } \\
\text { Serrat }\end{array}$ \\
\hline Feminino & $10 \%$ & $21 \%$ & $1 \%$ & $11 \%$ & $14 \%$ & $21 \%$ & $4 \%$ \\
\hline $\begin{array}{l}\text { Masculino } \\
\text { Prefiro não } \\
\text { informar }\end{array}$ & $0 \%$ & $7 \%$ & $0 \%$ & $2 \%$ & $5 \%$ & $4 \%$ & $0 \%$ \\
\hline
\end{tabular}

Fonte: Resultado da pesquisa (Elaborado pelos autores).

Os usuários do serviço público com formação educacional em Ensino Médio, das raças branca (19\%) e parda (21\%), são os principais pacientes do serviço de saúde bucal. A população com formação educacional em Ensino Fundamental, os usuários das raças negra e parda são os principais beneficiários do serviço de saúde bucal no município. 
Tabela 13 - Proporção entre a raça e a escolaridade

\begin{tabular}{|c|c|c|c|c|}
\hline \multirow[b]{2}{*}{ Raça } & \multicolumn{4}{|l|}{ Escolaridade } \\
\hline & $\begin{array}{l}\text { Ensino } \\
\text { Fundamental }\end{array}$ & $\begin{array}{l}\text { Ensino } \\
\text { Médio }\end{array}$ & $\begin{array}{l}\text { Ensino } \\
\text { Superior }\end{array}$ & $\begin{array}{l}\text { Prefiro não } \\
\text { informar }\end{array}$ \\
\hline Amarelo & $1 \%$ & $1 \%$ & $1 \%$ & $0 \%$ \\
\hline Branco & $8 \%$ & $19 \%$ & $4 \%$ & $0 \%$ \\
\hline Negro & $14 \%$ & $9 \%$ & $2 \%$ & $1 \%$ \\
\hline Pardo & $12 \%$ & $21 \%$ & $3 \%$ & $0 \%$ \\
\hline $\begin{array}{l}\text { Prefiro não } \\
\text { informar }\end{array}$ & $2 \%$ & $1 \%$ & $0 \%$ & $1 \%$ \\
\hline
\end{tabular}

Fonte: Resultado da pesquisa (Elaborado pelos autores).

A unidade Conjunto Luiz Alberto Argon não teve pacientes das raças amarela, branca e parda, enquanto as unidades Fonseca Almeida, Gulf e Mont Serrat não atenderam aos usuários da raça amarela. Na unidade Mont Serrat não foi registrada a presença de pacientes negros, como apresentado na Tabela 14.

Os pacientes da raça branca utilizam, em maioria, as unidades Centro, Fonseca Almeida e Gulf, enquanto os pacientes da raça negra, utilizam em maioria as unidades Gulf e Grotão. Já os usuários da raça parda são usuários das unidades Gulf e Centro.

Tabela 14 - Raça e as Unidades Básicas de Saúde

\begin{tabular}{|l|l|l|l|l|l|l|l|}
\hline \multicolumn{7}{|l|}{ Unidade Básica de Saúde } \\
\hline Raça & $\begin{array}{l}\text { Afonso } \\
\text { Arinos }\end{array}$ & Centro & $\begin{array}{l}\text { Conjunto Luiz } \\
\text { Alberto Argon }\end{array}$ & $\begin{array}{l}\text { Fonseca } \\
\text { Almeida }\end{array}$ & Grotão & Gulf & $\begin{array}{l}\text { Mont } \\
\text { Serrat }\end{array}$ \\
\hline Amarelo & $0 \%$ & $1 \%$ & $0 \%$ & $0 \%$ & $2 \%$ & $0 \%$ & $0 \%$ \\
\hline Branco & $4 \%$ & $9 \%$ & $0 \%$ & $7 \%$ & $5 \%$ & $5 \%$ & $2 \%$ \\
\hline Negro & $4 \%$ & $4 \%$ & $1 \%$ & $4 \%$ & $7 \%$ & $8 \%$ & $0 \%$ \\
\hline Pardo & $2 \%$ & $12 \%$ & $0 \%$ & $2 \%$ & $6 \%$ & $12 \%$ & $2 \%$ \\
\hline
\end{tabular}




\begin{tabular}{|l|l|l|l|l|l|l|l|}
\hline $\begin{array}{l}\text { Prefiro } \\
\text { não } \\
\text { informar }\end{array}$ & $1 \%$ & $2 \%$ & $0 \%$ & $0 \%$ & $0 \%$ & $0 \%$ & $0 \%$ \\
\hline
\end{tabular}

Fonte: Resultado da pesquisa (Elaborado pelos autores).

A Tabela 15 demonstra usuários com Ensino Superior estão distribuídos na maioria das unidades básica, exceto nos Conjunto Luiz Alberto Argon e Mont Serrat. A unidade Centro concentra a maioria dos usuários com Ensinos Fundamental e Médio.

Tabela 15 - Escolaridade e as Unidades Básicas de Saúde

\begin{tabular}{|l|l|l|l|l|l|l|l|}
\hline \multicolumn{7}{|l|}{ Unidade Básica de Saúde } \\
\hline & $\begin{array}{l}\text { Afonso } \\
\text { Arinos }\end{array}$ & $\begin{array}{l}\text { Centro } \\
\text { Escolaridade }\end{array}$ & $\begin{array}{l}\text { Conjunto } \\
\text { Luiz } \\
\text { Alberto } \\
\text { Argon }\end{array}$ & $\begin{array}{l}\text { Fonseca } \\
\text { Almeida }\end{array}$ & Grotão & Gulf & $\begin{array}{l}\text { Mont } \\
\text { Serrat }\end{array}$ \\
\hline $\begin{array}{l}\text { Ensino } \\
\text { Fundamental } \\
\text { Ensino Médio }\end{array}$ & $4 \%$ & $8 \%$ & $0 \%$ & $3 \%$ & $8 \%$ & $12 \%$ & $2 \%$ \\
\hline $\begin{array}{l}\text { Ensino } \\
\text { Superior }\end{array}$ & $2 \%$ & $19 \%$ & $1 \%$ & $6 \%$ & $8 \%$ & $10 \%$ & $2 \%$ \\
\hline $\begin{array}{l}\text { Prefiro não } \\
\text { informara }\end{array}$ & $0 \%$ & $0 \%$ & $0 \%$ & $2 \%$ & $0 \%$ & $0 \%$ & $0 \%$ \\
\hline
\end{tabular}

Fonte: Resultado da pesquisa (Elaborado pelos autores).

Foram apresentadas as tabelas dos resultados no intuito de facilitam a visualização dos dados e no próximo capítulos são apresentadas as discussões.

\section{DISCUSSÕES}

A literatura demonstra a existência de técnicas de análises estatísticas avançadas que podem fornecer resultados com outro nível de acurácia, no entanto, a simplicidade do 
tratamento e análise dos dados por meio da Estatística descritiva pode se mostrar eficiente em municípios que carência de profissionais com formações para emprego de técnicas robustas da área de exatas.

A pesquisa com abordagem quantitativa permite a generalização a partir dos dados e isso pode representar uma limitação aos resultados. Desta forma, os estudos que empreguem técnicas da abordagem qualitativa aplicada a grupos específicos podem trazer novos resultados.

Compreender o perfil dos usuários dos serviços públicos por si só não garante o acesso a tais serviços, mas permite que os gestores públicos e de saúde possam traçar estratégicas regionalizadas e que atenda às necessidades e características específicas de diversas populações.

Dados estimados do INCA indicam o surgimento de casos novos de câncer da cavidade oral esperados para o Brasil, para cada ano do triênio 2020-2022. Para o referido instituto, serão 11.180 casos em homens e de 4.010 em mulheres.

Os dados da pesquisa demonstraram que adesão aos serviços de saúde bucal por pessoas do sexo masculino no município de Comendador Levy Gasparian é superior ao feminino, seguindo a contramão dos dados estimados do INCA.

\section{CONCLUSÕES}

A pesquisa se dedicou a identificar o perfil dos usuários dos serviços de saúde bucal do município de Comendador Levy Gasparian, situado no interior do Estado do Rio de Janeiro. Para tal, foi aplicado um questionário para identificar aspectos demográficos dos usuários quanto a faixa etária, gênero, raça, escolaridade e unidade básica de saúde utilizada.

No aspecto relativo à faixa etária, os resultados indicaram um acesso menor ao serviço por parte das pessoas idosas e mais jovens. Em relação a população jovem, ressalta-se que a pesquisa se restringiu aos usuários com mais de 18 anos de idade em virtude na necessidade de autorização de responsáveis para participação da 
pesquisa, tal fato se demonstra uma limitação para resultados apurados que não considerou a potencialidade de todas as faixas etárias do público jovem.

Estratégias que sensibilizem pessoas idosas e jovens quanto aos cuidados com a saúde bucal podem ser oportunas no município.

Em relação ao gênero, a presença de mulheres se apresentou muito menor que a presença de homens. Tal resultado pode indicar a necessidade de ações públicas que atraiam para tratamento odontológico no município.

Quanto a raça, os resultados mostraram uma distribuição equitativa entre brancos, negros e pardos. Destacam-se a exceção relativa à quantidade de pessoas que se declararam da raça amarela e dos que preferiram não informar.

Os dados referentes à escolaridade dos usuários indicaram que a maioria possui Ensino Fundamental e Médio, públicos que tendem a se inserirem em atividades laborais com menores rendas e normalmente os que necessitam dos serviços de saúde pública.

As unidades básicas de saúde do Centro e próximas a região central do município (Grotão e Gulf) tem um número de acesso aos serviços de saúde bucal superior quando comprado às unidades mais afastadas.

Unidades básicas de saúde que se encontram distantes da região central podem buscar estratégias de sensibilização dos usuários durante as ações da visita da Saúde da Família.

Recomenda-se como pesquisa futuras o uso de técnicas de análises com uma abordagem quantitativa mais complexas: séries temporais, regressões e outras.

Estudos que busquem analisar o perfil dos usuários dos serviços de saúde bucal no grupo de adolescentes, crianças, assim como em unidades escolares e prisionais. 


\section{REFERÊNCIAS}

ARAÚJO-JÚNIOR, Júlio Leite de et al. Perfil clínico e epidemiológico das alterações bucais em portadores do HIV. Archives of Health Investigation, v. 7, n. 8, p. 339$343,2018$.

BÊRNI, Duilio de Avila; FERNANDEZ, Brena Paula Magno. Métodos e Técnicas de Pesquisa: Modelando as ciências empresariais. São Paulo: Saraiva, 2012.

BRASIL. Ministério da Saúde. Decreto ㄲo 9.795, de 17 de maio de 2019. Aprova a Estrutura Regimental e o Quadro Demonstrativo dos Cargos em Comissão e das Funções de Confiança do Ministério da Saúde, remaneja cargos em comissão e funções de confiança, transforma funções de confiança e substitui cargos em comissão do Grupo-Direção e Assessoramento Superiores - DAS por Funções Comissionadas do Poder Executivo - FCPE. Brasília: Ministério da Saúde, 2019. Disponível em: <http://www.planalto.gov.br/ccivil_03/_ato20192022/2019/decreto/D9795.htm>. Acesso em 10 nov. 2019.

BRASIL. Ministério da Saúde. Passo a passo das ações do Brasil Sorridente. Brasília: Ministério da Saúde; 2013.

BRASIL. Ministério da Saúde. Diretrizes da política nacional de saúde bucal. Brasília: Ministério da Saúde; 2004.

CAMPOS, Fernanda Araújo Trigueiro et al. Manifestações bucais decorrentes da quimioterapia em crianças. Revista Campo do Saber, v. 4, n. 5, p. 136-159, 2018.

CRESWELL, John W.. Projetos de Pesquisa: métodos qualitativo, quantitativo e mistos. Porto Alegre: Artmed; 2010.

FERREIRA, Denise Carvalho et al. Aspectos psicossociais e percepção de impacto da saúde bucal na qualidade de vida em adultos do Sul do Brasil. Revista Brasileira de Epidemiologia, v. 23, e200049, p. 1-13, 2020. 
GIOVANELLA, Lígia et al. De Alma-Ata a Astana. Atenção primária à saúde e sistemas universais de saúde: compromisso indissociável e direito humano fundamental. Caderno de Saúde Pública, v. 35, n. 3, e00012219, 2019.

GUERRA, Letícia de Freitas Cuba; SANTOS, Ana Tayline do. Perfil odontológico de pacientes internados na Unidade de Terapia Intensiva de um hospital oncológico do Sudoeste do Paraná. Arquivos de Ciências da Saúde da UNIPAR, v. 22, n. 2, p. 75 80, 2018.

INSTITUTO NACIONAL DE CÂNCER (INCA). Sistema de Informações sobre Mortalidade. Atlas On-line de Mortalidade. Disponível em: https://mortalidade.inca.gov.br/MortalidadeWeb/pages/Modelo10/consultar.xhtml. Acesso em 03 jan. 2020.

JUCÁ, Maria Augusta Cunha Lima et al. Impactos da saúde bucal de usuários com HIV/Aids em um serviço especializado. Revista Brasileira de Enfermagem, v. 72, n. 6, p. 1649-1657, 2019.

MINISTÉRIO DA SAÚDE. Estimativa 2020: incidência de câncer no Brasil. Rio de Janeiro: INCA; 2019. 120 p.

MINISTÉRIO DA SAÚDE. Ministério da Saúde chama atenção para a Semana Nacional de Prevenção do Câncer Bucal [Internet]. Brasil: Ministério da Saúde; 05/11/2018 [revisado em 05/11/2018]. Disponível em: https://www.saude.gov.br/noticias/agencia-saude/44603-ministerio-da-saude-chamaatencao-para-a-semana-nacional-de-prevencao-do-cancer-bucal. Acesso em 10 nov. 2019.

MINISTÉRIO DA SAÚDE. Portaria de Consolidação ํำ 2, de 28 de setembro de 2017. Consolidação das normas sobre as políticas nacionais de saúde do Sistema Único de Saúde. Brasília: Ministério da Saúde, 2017. Disponível em: <http://bvsms.saude.gov.br/bvs/saudelegis/gm/2017/prc0002_03_10_2017.html>. Acesso em 11 nov. 2019. 
MINISTÉRIO DA SAÚDE. Portaria oㅡ 2.436, de 21 de setembro de 2017. Aprova a Política Nacional de Atenção Básica, estabelecendo a revisão de diretrizes para a organização da Atenção Básica, no âmbito do Sistema Único de Saúde (SUS). Brasília: Ministério da Saúde, 2017. Disponível em: <http://bvsms.saude.gov.br/bvs/saudelegis/gm/2017/prc0002_03_10_2017.html>. Acesso em 11 nov. 2019.

OLIVEIRA, Eduardo José Pereira et al. Qualidade de vida e condições de saúde bucal de hipertensos e diabéticos em um município do Sudeste Brasileiro. Ciência \& Saúde Coletiva, v. 23, n. 3, p. 763-772, 2018.

ORGANIZAÇÃO DAS NAÇÕES UNIDAS (ONU). Transformando Nosso Mundo: A Agenda 2030 para o Desenvolvimento Sustentável. 2015. 49 p. Disponível em: <https://nacoesunidas.org/wp-content/uploads/2015/10/agenda2030-pt-br.pdf>. Acesso em: 15 nov. 2019.

ORGANIZAÇÃO DAS NAÇÕES UNIDAS (ONU). Declaração Universal dos Direitos Humanos. 2009. 10 p. Disponível em: <https://nacoesunidas.org/wpcontent/uploads/2018/10/DUDH.pdf>. Acesso em: 14 nov. 2019.

PERES, Karen Glazer et al. Redução das desigualdades sociais na utilização de serviços odontológicos no Brasil entre 1998 e 2008. Revista de Saúde Pública, v. 46, n. 2, p. 250-258, 2012.

SANTOS, Isabela Vieira et al. Práticas Acadêmicas, Cirurgiões-dentistas, População e Câncer Bucal. Revista Odontologia Clínico-Científica, v. 10, n. 3, p. 207-210, 2011.

SLADE, Gary D.; SPENCER, A. J. Development and evaluation of the Oral Health Impact Profile. Community Dental Health, v. 11, n. 1, p. 3-11, 1994.

SOUZA, Anderson Jambeiro de et al. Factors associated with dental caries, periodontitis and intra-oral lesions in individuals with HIV / AIDS. Journal AIDS Care, v. 30, n. $5,2018$. 
TERRA, Betina Garay; GOULART, Ricardo Rahal; BAVARESCO, Caren Serra. O cuidado odontológico do paciente portador de diabetes mellitus tipo 1 e 2 na Atenção Primária à Saúde. Revista APS, v. 14, n. 2, p. 149-161, 2011.

WORLD HEALTH ORGANIZATION (WHO). Oral health surveys: basic methods. World Health Organization; 2013. 142 p.

Enviado: Setembro, 2020.

Aprovado: Outubro, 2020. 